\title{
AC 2010-1764: FROM REMEDIATION TO APPLICATION: AN INVESTIGATION OF COMMON MISCONCEPTIONS ASSOCIATED WITH VECTOR ANALYSIS IN AN UNDERGRADUATE BIOMECHANICS COURSE
}

\section{Sara Koehler, Northwestern University}

SARA R. KOEHLER is a Ph.D. candidate in the Biomedical Engineering Department at Northwestern University and a participant in the Graduate Teaching Certificate Program at Northwestern University's Searle Center for Teaching Excellence. Her research focuses on the biomechanics of transfemoral amputee gait.

\section{Wendy Murray, Northwestern University}

WENDY M. MURRAY is an Assistant Professor at Northwestern University, with joint appointments in Biomedical Engineering in the Robert R. McCormick School of Engineering and Applied Science, and in Physical Medicine and Rehabilitation in the Feinberg School of Medicine. She teaches both undergraduate and graduate level biomechanics classes. Her research focuses on using biomechanics as a framework for understanding how we move and control our arms and hands. 


\title{
From Remediation to Application: An Investigation of Common Misconceptions Associated with Vector Analysis in an Undergraduate Biomechanics Course
}

\begin{abstract}
Introduction to Biomechanics (BME 271) is a required course in the Department of Biomedical Engineering at Northwestern University, covering basic concepts in rigid-body mechanics with applications in biology and physiology. Building on previous experience with vector analysis, Newtonian mechanics, and free-body diagrams, BME 271 is designed to provide sophomore engineering students with a foundation in statics and strength of materials in preparation for more advanced topics in dynamics and soft-tissue mechanics. In order to promote student engagement and knowledge transfer within our curriculum, we have recently incorporated several innovative teaching methods into our instruction base, including online courseware developed by the VaNTH ERC (Vanderbilt University; Northwestern University; University of Texas at Austin; and Health, Science and Technology at Harvard/MIT Engineering Research Center) for Bioengineering Educational Technologies ${ }^{1}$, Personal Response Systems (PRS) to enhance formative assessment, and challenge-based homework assignments to emphasize the application of fundamental engineering skills in biomechanics. The goal of this paper is to discuss our experience with these methods, highlighting how we have used PRS to systematically diagnose and address common misconceptions associated with prerequisite course material and guide our delivery of new concepts in order to improve learning outcomes.
\end{abstract}

\section{Introduction}

Over the past twenty years, undergraduate education in the field of biomedical engineering (BME) has undergone a period of rapid growth. Fueled partially by institutional grants made available by the Whitaker Foundation in the early 1990s, ABET-accredited BME programs have increased in number from 24 in $2003^{2}$ to 60 in $2008^{3}$. At the same time, technological advances have inspired tremendous diversity among these programs, exposing today's BME students to a broader range of curricular objectives. To establish a strong program identity and to ensure that BME students share a common knowledge base, academic leaders have recently turned their attention toward developing a core undergraduate BME curriculum ${ }^{2-5}$. Among several recommended courses, biomechanics has been identified as an essential sub-discipline within a BME student's education ${ }^{6-8}$.

At Northwestern University, Introduction to Biomechanics (BME 271) is a required course for all BME undergraduates. Compared to more conventional mechanics courses offered through sister departments, the goal of BME 271 is to anchor fundamental concepts in statics and strength of materials within the context of biology and physiology. Beginning in 2008, the instructional focus of BME 271 has been to replace traditional, lecture-based methods of teaching with a more challenge-driven approach. According to studies conducted by the VaNTH $\mathrm{ERC}^{8,9}$, challenge-driven strategies promote active learning by creating environments that are learner-centered, knowledge-centered, assessment-centered, and community-centered ${ }^{10}$. Since the introduction of these methods, we contend that one of the most challenging aspects of teaching BME 271 is helping students to transfer basic skills in math and science to novel 
applications in biomechanics. Specifically, we have identified conceptual questions involving vector projections to be particularly problematic for many students, limiting their understanding of higher-level concepts that build on these skills.

In order to support applied skills in BME 271, we have implemented several methods to engage students in a process of review and assessment intended to diagnose and address common misconceptions associated with vector analysis. These methods include online courseware developed by the VaNTH ERC ${ }^{1}$, PRS to enhance formative assessment, and challenge-based homework assignments to emphasize the application of fundamental engineering skills in biomechanics. The goal of this paper is to discuss our experience with these methods, highlighting how we have used PRS to systematically diagnose and address common misconceptions associated with prerequisite course material and guide our delivery of new concepts in order to improve learning outcomes.

\section{Methods}

Three consecutive quarters of BME 271 were investigated in this study: Winter 2008 (W08), Winter 2009 (W09), and Winter 2010 (W10). Winter 2008 represents the senior author's (WMM) first experience in teaching BME 271; the junior author (SRK) served as a graduate teaching fellow in W09. Twenty-seven students were enrolled in W08 (9 sophomores, 15 juniors, and 3 seniors), 41 students were enrolled in W09 (1 freshman, 14 sophomores, 21 juniors, and 5 seniors), and 43 students were enrolled in W10 (17 sophomores, 18 juniors, and 8 seniors). Each class was comprised of mostly BME majors. Course prerequisites included experience with vector analysis, differentiation, integration, Newtonian mechanics, and free-body diagrams. Most students satisfied these prerequisites through a departmental course sequence titled Engineering Analysis.

\subsection{Adaptive Learning Technology System: Vector Analysis Tutorial}

At the beginning of each quarter (W08, W09, and W10), students completed an online vector analysis tutorial developed by the VaNTH ERC ${ }^{1}$. At the beginning of the tutorial, students were asked to complete a pre-test (Fig. 1), which included the following six questions:

Q1) Identify the X- and y-components of two vectors,

Q2) Subtract two vectors,

Q3) Calculate the magnitude and direction of a resultant vector,

Q4) Calculate the components of a unit vector,

Q5) Find the projection of one vector in the direction of another vector, and

Q6) Calculate the cross product of two vectors.

Students were not graded on their pre-test performance, however this exercise provided feedback (in this case, right vs. wrong) regarding mistakes/misconceptions and was intended to motivate further remediation efforts. Based on the poor performance of W08 students on subsequent inclass and exam assessments (see Results), pre-test results were saved in W09 and W10 so that short-term learning gains associated with this tutorial could be analyzed.

Following the pre-test, students completed a guided vector analysis tutorial. This tutorial was presented via an online PowerPoint ${ }^{\circledR}$ lecture, allowing students to control the pace of the 
presentation. As opposed to applied problems, vector analysis concepts were presented in a mathematical context. Students were allowed to stop and return to the tutorial at any time and they could review the material on multiple occasions. Following the tutorial, students completed a post-test, which paralleled the pre-test questions. However, initial conditions were randomized so that each student solved a slightly different version of the same problem. The results of the post-test were recorded for grading purposes. Students were given one chance to answer correctly before points were deducted from their total score.

Performance on individual pre-test questions were compared for W09 and W10 using a repeated measures ANOVA with Bonferonni adjustment $(\mathrm{p}<0.05)$. Significant differences between preand post-test performance were assessed using a paired t-test $(\mathrm{p}<0.05)$.
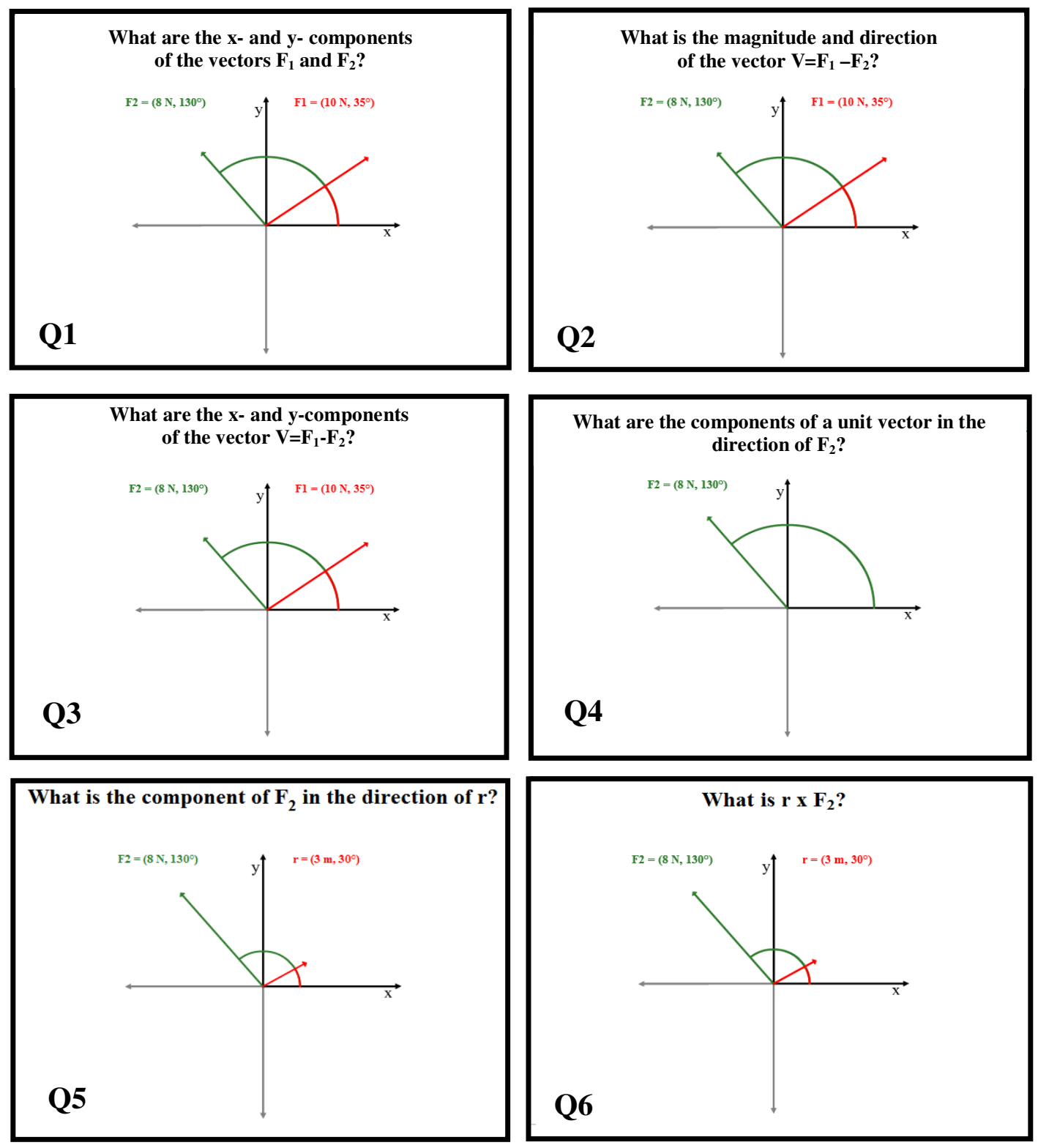

Figure 1: Pre-test vector tutorial questions. 


\subsection{In-Class Assessment: Personal Response Systems}

Recognizing that classroom interaction is an effective means to improve learning outcomes, PRS use has gained popularity in recent years as a communication tool in post-secondary institutions. Particularly valuable in disciplines that favor large class sizes, PRS units have been used with varying success in a wide variety of fields, including physics, chemistry, biology, economics, and management ${ }^{11-15}$. By using presentation software (such as PowerPoint ${ }^{\circledR}$ ), a remote transmitter device (assigned to individual or groups of students), a single receiver (connected to the instructor's laptop), and software that pools and presents the transmitted data, PRS units provide instructors with the ability to pose questions to students during a lecture and receive realtime feedback regarding their response.

In BME 271, PRS use was designed to provide diagnostic feedback, formative assessment, and the opportunity to address misconceptions in a timely manner. During the first week of the quarter, students registered their PRS device, enabling class participation to be monitored (5\% of total grade). Participation grades were based on PRS response frequency, not accuracy. In total, more than 40 PRS questions were presented throughout the quarter.

\subsubsection{Winter Quarter 2008 (W08)}

During W08, students were asked to solve a complicated PRS story problem involving vector projections (Fig. 2a). This question was posed during the lecture in which the online vector homework was due. That is, the students had completed the online vector tutorial, with a graded post-test, within 48 hours of the class period. With regard to the PRS story problem, students were asked to calculate the scalar projection of one vector in the direction of another vector and to select the correct answer among 6 possibilities. These possibilities incorporated common mistakes, including sign errors, finding the vector's magnitude instead of its projection, and interpreting the vector projection to be in the wrong quadrant. In order to assess perceived skill, W08 students were also asked in a later class to indicate whether they knew how to calculate the component of one vector in the direction of another vector (Fig. 2b).

a)

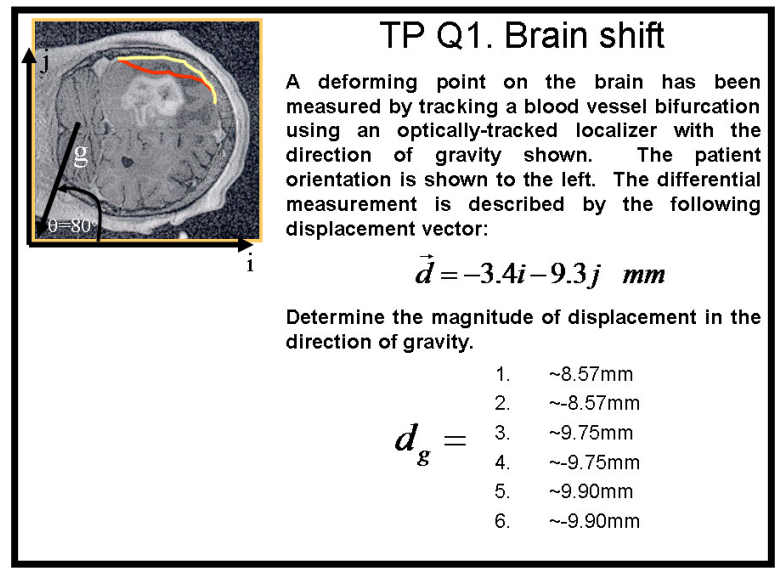

b)

TP Q2. I know how to calculate the
component of one vector in the
direction of another vector
1. True
2. False

Figure 2: PRS questions presented during W08. 


\subsubsection{Winter Quarter 2009 (W09)}

During W09, students were challenged with a similar applied PRS story problem (Fig. 3c), however, this story problem was presented two class periods after the online vector homework was due. In the first class following the vector homework, two (diagnostic) PRS questions were presented in order to identify common misconceptions involving vector projections. The first question required students to identify the correct formula to calculate the scalar projection of one vector onto another (Fig. 3a). Then, students were asked to solve a vector projection problem similar to Q5 (Fig. 1) on the online vector homework (Fig. 3b). In the following lecture, students completed the PRS story problem, which was re-worded slightly from W08 (Fig. 3c).

a)

TP Q1. In order to calculate the scalar projection of a vector $\mathbf{A}$ onto a vector $B$, you would use:

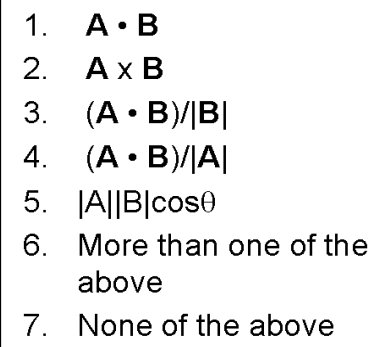

7. None of the above

c)

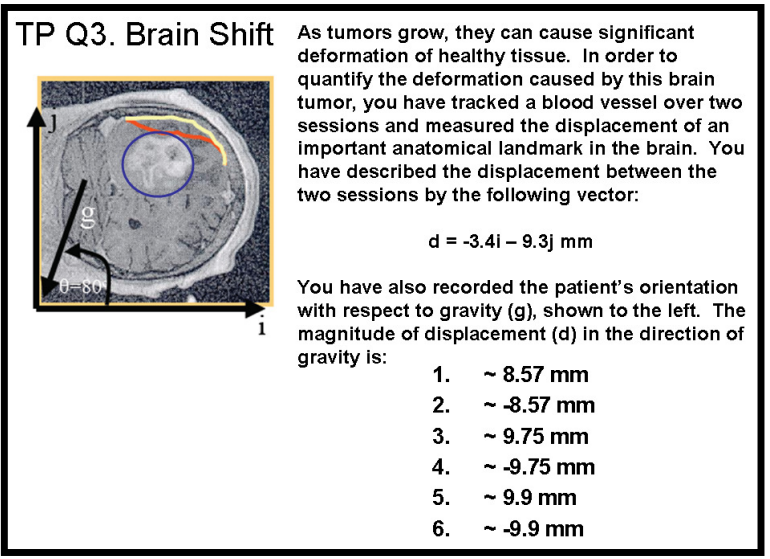

b)

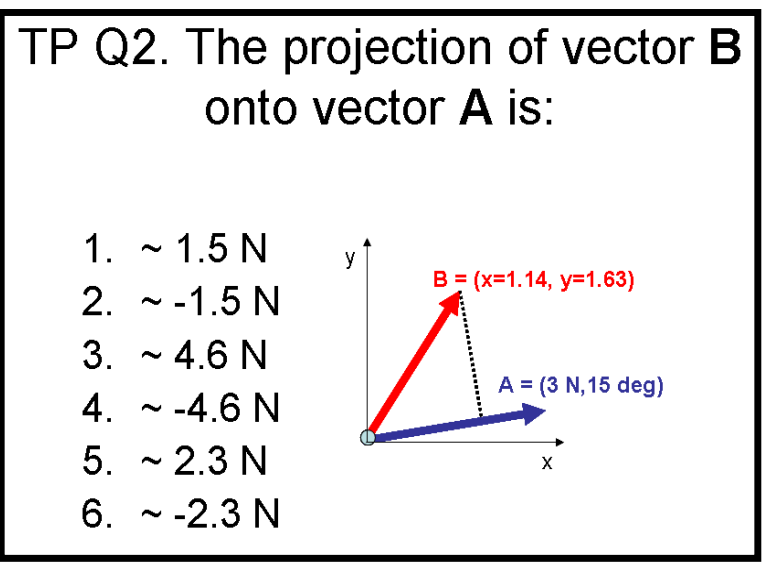

Figure 3: PRS questions presented during W09.

\subsubsection{Winter Quarter 2010 (W10)}

During W10, the same approach to PRS questions was implemented: in the first class period following the vector homework, basic diagnostic PRS questions were presented; in the second class period following the homework assignment, students were challenged with the same PRS 
story problem from W09 (Fig. 3c). In W10, three diagnostic questions were asked instead of two. Like W09, the first question required students to identify the correct formula to calculate the scalar projection of one vector onto another (Fig. 3a). Also like W09, students were asked to solve a vector projection problem similar to the one presented in the online vector analysis tutorial (Fig. 4a). Finally, W10 students were asked to identify the quadrant of the resulting projection (Fig. 4b).

a)

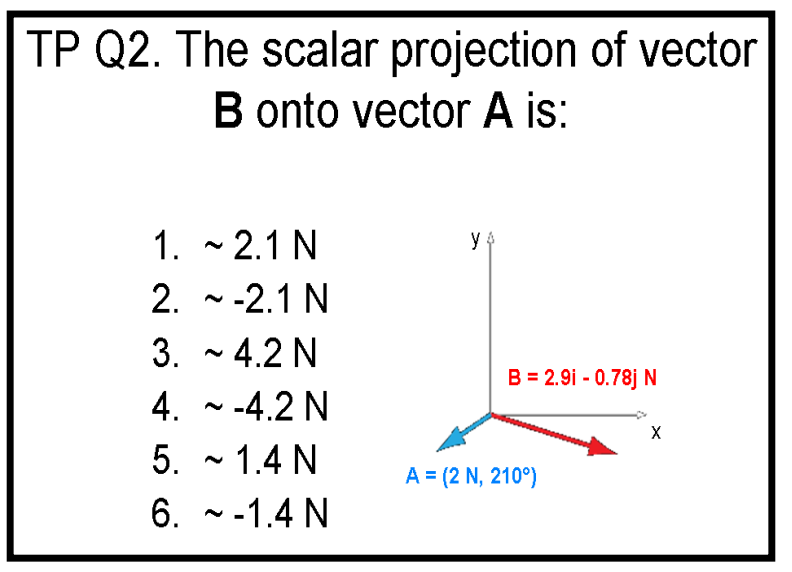

b)

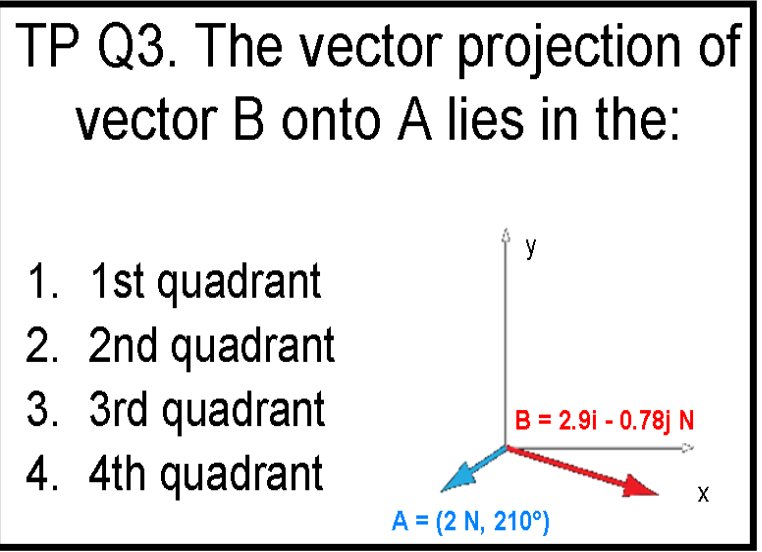

Figure 4: PRS questions presented during W10.

\subsection{Application-Driven Homework}

During W10, students were assigned an application-driven homework assignment, which called on students to recognize the application of vector projection skills in a novel biomechanics problem. Students were assigned the problem two weeks after completing the online vector tutorial homework, and the material being covered in class at that time was not directly related to the problem. Students were informed that the goal of the assignment, titled "Reality Check", was to give them experience assessing a real-world problem and deciding how to approach it. Students were asked to solve the problem independently, without input from others (including fellow students, the professor, or the TA), and were required to sign an honor statement indicating that they had done so. The grading structure was such that students could only be rewarded for deriving the correct answer, and they suffered no penalties for taking an incorrect approach.

In the problem, a scenario was described in which the student was summoned to serve as an expert consultant to provide their opinion regarding whether wrist guards should be redesigned by a manufacturing company. The manufacturing company was being sued by a snowboarder who had fractured his distal radius while wearing the wrist guards. The problem statement included "statements" from an orthopaedic surgeon, who described the fracture as being consistent with a uniaxial load and "concluded" that the fracture resulted from a compressive force acting along the longitudinal axis of the radius. An important component of class in the weeks preceding the assignment was a challenge-based series of lectures describing how a 
vertebrae would fail during uniaxial compression. The problem statement also included "results" from an accident reconstruction performed by an engineering consultant (Fig. 5).

The goal of the assignment was for the students to recognize that they could calculate the load applied along the longitudinal axis of the radius by performing a vector projection of the force in the direction of the forearm. The problem was designed so that if the students performed the vector projection correctly, they would conclude that the performance of the wrist guards would protect the radius from fracture. In contrast, if they performed the vector projection incorrectly, they would conclude the wrist guards did not protect the radius from fracture. The incorrect answer resulted from inaccurate identification of the angle between the two vectors $\left(65^{\circ} \mathrm{vs}\right.$. $115^{\circ}$ ) and/or inaccurate interpretation of the quadrant of the resulting vector projection (QI vs. QIII). Overall, the problem was intended for the students to implement a vector projection correctly, and to realize that the loading description provided by the engineering consultant did not correspond with the loading pattern believed by the surgeon to have caused the fracture.

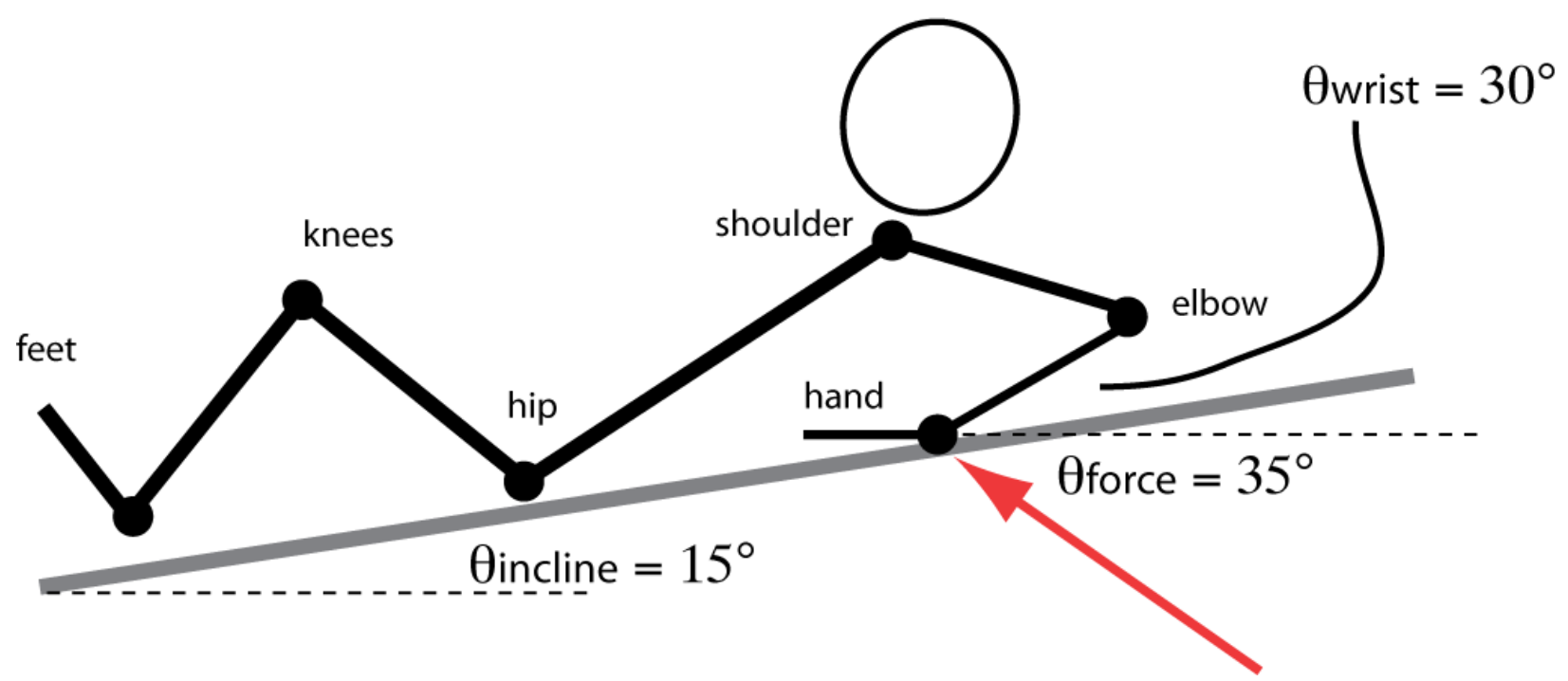

Figure 5: Diagram provided to students as a part of the accident reconstruction in the "Reality Check" problem.

\subsection{Learning Outcome: Midterm Exam}

\subsubsection{Winter Quarter 2009 (W09)}

In order to investigate learning gains associated with our intervention efforts during W09, students were presented with an applied problem on the midterm exam (Fig. 6). The goal of this question was two-fold: the first part of this question tested the students' ability to recognize a vector projection operation that was embedded within a story problem, thereby emphasizing transfer (i.e., the ability of students to apply their understanding of vector projections in a novel situation). The second part of the question evaluated the students' ability to interpret the results of their calculation, thereby emphasizing conceptual understanding. A common mistake observed during W08 was for students to assume that the scalar projection of one non-unit vector 
onto another non-unit vector was simply a dot product. During W09, PRS questions were designed to address this misconception. The exam problem in W09 was therefore written to assess whether remediation associated with this misconception improved learning outcomes.

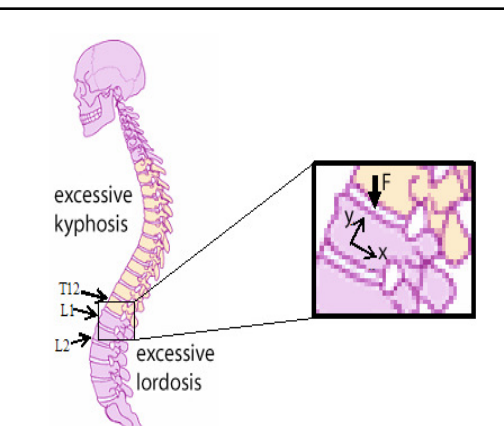

Kyphosis and lordosis are terms used to describe normal curvatures of the spine. When these curvatures are excessive, segmental loading patterns of the vertebra may be altered. As a biomedical engineer, you are interested in characterizing the altered load on a particular vertebra (L1) for someone with excessive lordosis. You establish a coordinate system for the vertebra, shown to the left.

The longitudinal axis of the vertebra is described by a vector:

$$
\vec{y}=2 \hat{i}+2 \hat{j}
$$

The transverse axis of the vertebra is described by a vector:

$$
\vec{x}=2 \hat{i}-2 \hat{j}
$$

A load is applied to L1 by the superior vertebra (T12) equal to:

$$
\vec{F}=-350 \hat{j} \mathrm{~N}
$$

g) Find the component of force $\vec{F}$ in the direction of the longitudinal axis of vertebra. Show your work.

h) Is the component of $\vec{F}$ along the long axis of the vertebra directed toward T12 or L2?

Figure 6: W09 midterm exam question.

\subsubsection{Winter Quarter 2009 (W09)}

Similar to W09, the W10 midterm exam included an applied vector projection problem (Fig. 7). The goals of the W10 exam problem remained the same; the question was designed to test the students' ability to recognize the application of a basic vector projection skill in an unfamiliar biomechanics problem. 
Prosthetic components are designed to withstand a certain amount of force. They are rated according to a user's body weight and activity level. As a biomedical engineer, you are conducting a quality assurance test to confirm that a new kind of prosthetic pylon is strong enough to support the ground reaction forces (GRFs) generated by a typical user at a range of walking speeds. In order to be approved for mass production, the pylon must comply with a safety factor of at least 1.4 for all walking conditions.

You are only concerned about the forces that develop along the longitudinal axis of the pylon because the prosthetic foot is designed to prevent transmission of force to the transverse axis of the pylon.

The material used in the pylon is isotropic. The pylon has a cross-sectional area of $5 \mathrm{~cm}^{2}$. Load-elongation tests performed with compressive forces suggest that pylons of the same dimensions consistently fail when $1550 \mathrm{~N}$ of force is applied along its longitudinal axis.

The figure to the right shows the orientation of pylon shortly after heel strike. The transverse and longitudinal axes of the pylon are described by the vectors:

$$
\begin{aligned}
& \vec{x}_{\text {pylon }}=8 \hat{i}+4.5 \hat{j} \\
& \vec{y}_{\text {pylon }}=-4.5 \hat{i}+8 \hat{j}
\end{aligned}
$$

You have also measured the vertical $\left(\mathrm{y}_{\mathrm{GRF}}\right)$ and horizontal $\left(\mathrm{x}_{\mathrm{GRF}}\right)$ ground reaction forces generated by a typical user shortly after heel strike for range of walking speeds:

\begin{tabular}{|c|c|c|}
\hline $\begin{array}{c}\text { Walking } \\
\text { Speed (m/s) }\end{array}$ & $\begin{array}{c}\text { Vertical } \\
\text { GRF (N) }\end{array}$ & $\begin{array}{c}\text { Horizontal } \\
\text { GRF (N) }\end{array}$ \\
\hline 0.6 & 950 & 100 \\
\hline 1.4 & 1200 & 250 \\
\hline 2.1 & 1600 & 650 \\
\hline
\end{tabular}

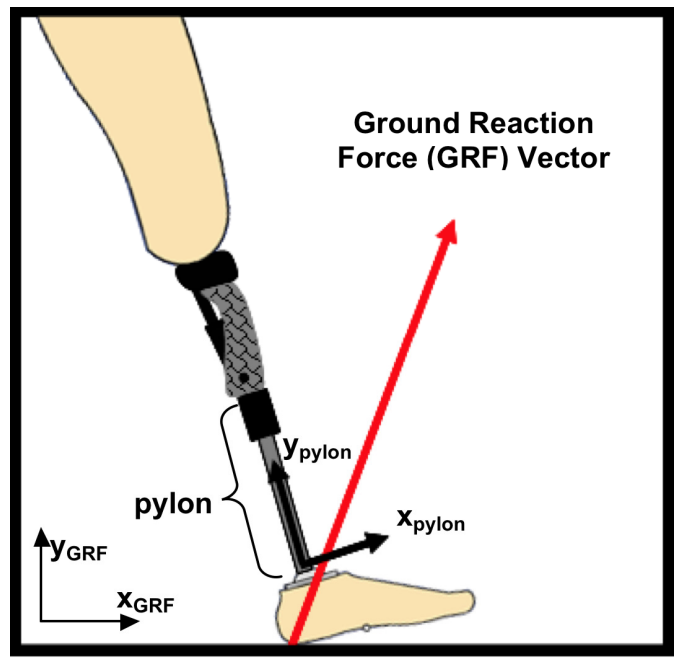

\section{A) What is the magnitude of the GRF along the longitudinal axis of the pylon for a walking speed of $2.1 \mathrm{~m} / \mathrm{s}$ ? Please show your work.}

Figure 7: W10 midterm exam question.

\section{Results}

\subsection{Adaptive Learning Technology System: Vector Analysis Tutorial}

As described in Section 2.1, students were asked to complete a series of six pre-test questions (Q1-Q6). Results from W09 and W10 indicate that prior to review, students consistently demonstrated low-level competency on Q5, which assessed their ability to calculate the scalar 
projection of one vector in the direction of another non-unit vector (Fig. 8). During both W09 and W10, less than 50\% of the class was able to successfully execute this skill. During W09, Q5 performance was significantly lower than several other pre-test questions, including Q1 (the ability to identify the $\mathrm{x}$ - and $\mathrm{y}$-components of two vectors; $\mathrm{p}=0.05$ ), Q2 (the ability to subtract two vectors; $\mathrm{p}=0.005$ ), and $\mathrm{Q} 3$ (the ability to calculate the magnitude and direction of a resulting vector; $\mathrm{p}<0.001)$. During W10, Q5 performance was significantly lower than Q3 ( $<<0.001)$.

a)

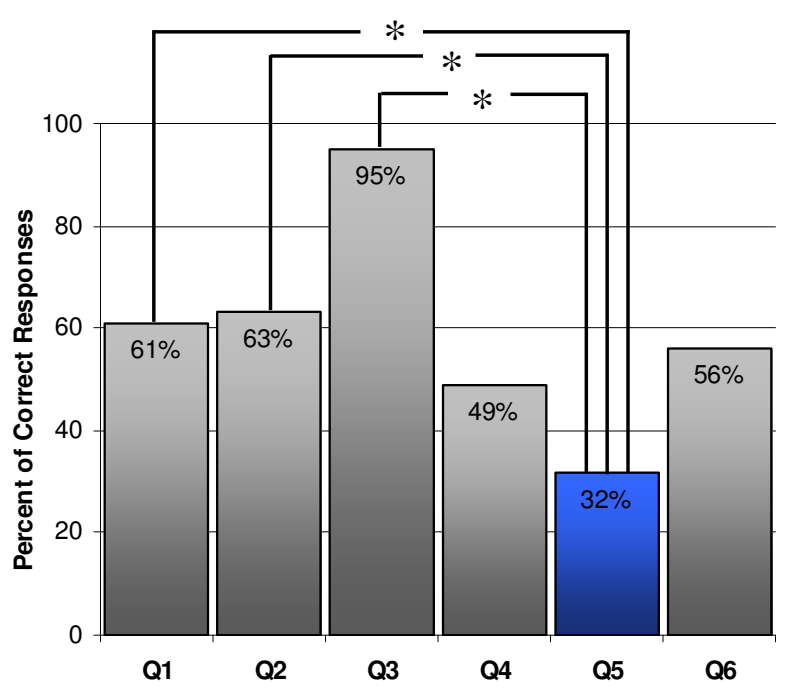

b)

W10 Pre-Test

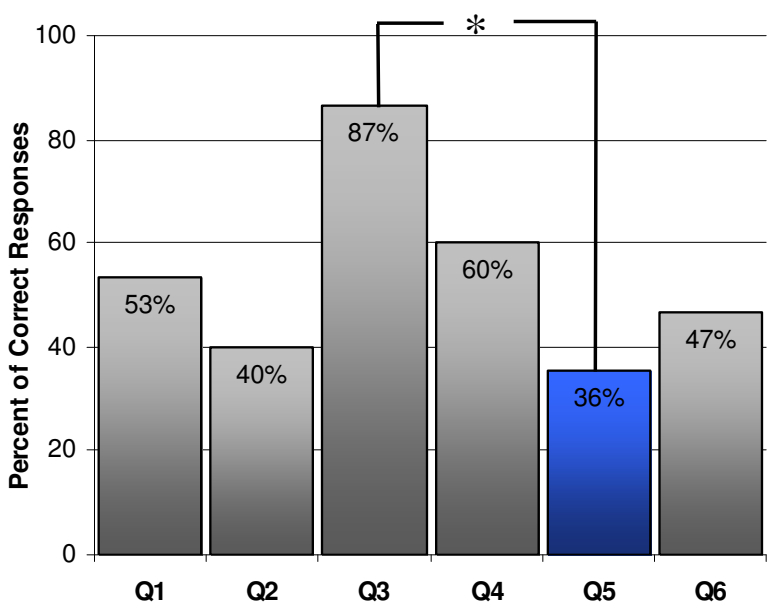

Figure 8: Pre-test results from the online vector analysis tutorial, which reflect baseline competency prior to review. Students demonstrated low-level performance in vector projection skills, shown in blue.

a)

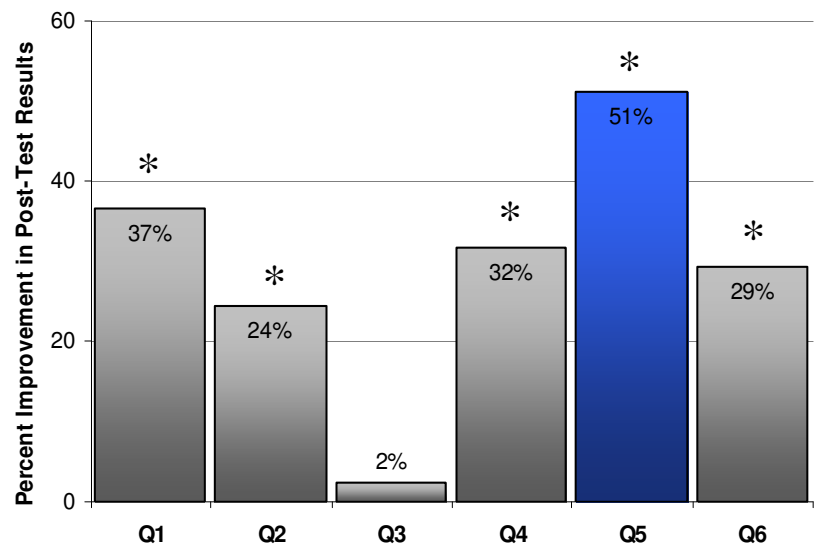

b)

W10 Post-Test Improvement

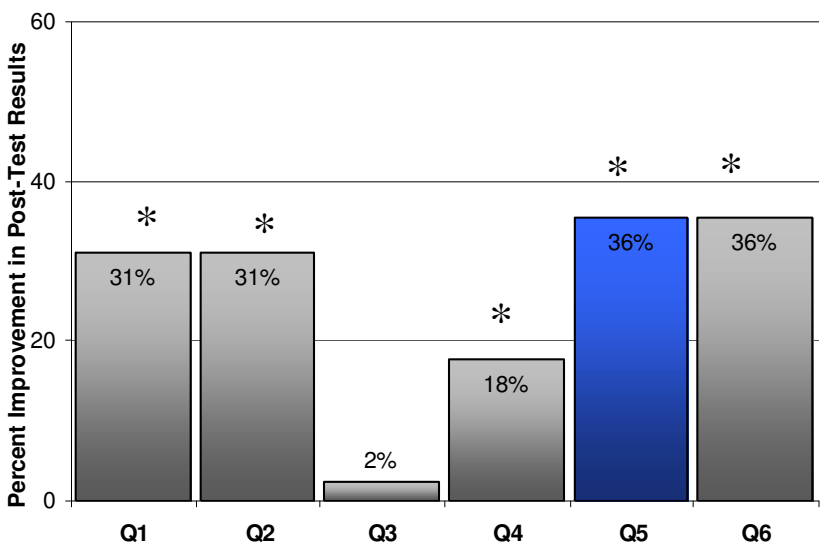

Figure 9: Learning gains associated with the vector analysis tutorial, defined as the difference between average pre- and post-test scores. Asterisk indicates significant improvement in posttest performance. Largest improvements were observed in vector projections, shown in blue. 
Following a self-paced, online review of vector algebra, students demonstrated a significant improvement in nearly all post-test questions (Fig. 9). During W09, the largest learning gain involved Q5 (51\% improvement, $\mathrm{p}<0.001)$. During W10, students demonstrated equally large learning gains for Q5 (36\% improvement, p<0.001) and Q6 (36\% improvement, p<0.001). Significant improvement, demonstrated in nearly all post-test questions, indicates considerable learning gains in a relatively short period of time.

\subsection{In-Class Assessment: Personal Response Systems}

\subsubsection{Winter Quarter 2008 (W08)}

After completing the online vector analysis tutorial, W08 students were presented with an applied PRS question during the following lecture. This question was designed to test the students' ability to find the scalar projection of one vector in the direction of another vector, as this skill is important for subsequent material involving stress transformations and equivalent force systems.

Surprisingly, only $20 \%$ of students answered this question correctly, despite having completed the online vector homework (for a grade) within 48 hours of the class period. Table 1, which summarizes these results, shows that the most common mistake in W08 involved students solving for the magnitude of $\vec{d}$ instead of the scalar projection of $\vec{d}$ in the direction of $\vec{g}$. Concerned that insufficient preparation in vector algebra would hinder progress in subsequent material in stress transformations, additional lecture time was spent explaining vector projections. A pop quiz was given to students during the following lecture which required students to find the scalar projection of one vector onto another; however unlike the PRS question, this skill was not embedded with a story problem. Students performed slightly better on this pop quiz (38\% percent answered the question correctly), however a majority of the class still did not demonstrate competency in this skill.

Table 1: Response distribution for PRS story problem (Figs. 2a and 3c).

\begin{tabular}{|c|c|c|c|c|}
\hline Answers & W08 & W09 & W10 & Mistake \\
\hline 1) $8.57 \mathrm{~mm}$ & $8 \%$ & $10 \%$ & $10 \%$ & Sign error in Part 1 \\
\hline 2) $-8.57 \mathrm{~mm}$ & $12 \%$ & $6 \%$ & $0 \%$ & Sign error in Part 2 \\
\hline 3) $9.75 \mathrm{~mm}^{\star}$ & $20 \%$ & $35 \%$ & $44 \%$ & None \\
\hline 4) $-9.75 \mathrm{~mm}$ & $4 \%$ & $26 \%$ & $10 \%$ & Assumed g was in $1^{\text {st }}$ quadrant (instead of $3^{\text {rd }}$ ) \\
\hline 5) $9.9 \mathrm{~mm}$ & $28 \%$ & $13 \%$ & $21 \%$ & Found magnitude of $d$ (instead of projection) \\
\hline 6) $-9.9 \mathrm{~mm}$ & $28 \%$ & $10 \%$ & $15 \%$ & Found magnitude of $d$ and made a sign error \\
\hline
\end{tabular}

*Solution: $\frac{(\vec{d} \bullet \vec{g})}{|\vec{g}|}=(\underbrace{(-3.4) *(-\cos 80)})+\underbrace{(-9.3) *(-\sin 80)})=0.59+9.16=9.75 \mathrm{~mm}$

Part 1

Part 2

Interestingly, when explicitly asked whether they understood how to calculate the scalar projection of one vector in the direction of another vector, $91 \%$ of the class believed that they 
were able to perform this skill, demonstrating a discrepancy between perceived and actual skill level. After viewing the results of the PRS true/false question, students were presented with the results of the pop quiz, in an attempt to help them realize that they needed to improve their vector analysis skills.

\subsubsection{Winter Quarter 2009 (W09)}

Based on observations during W08, we extended our use of PRS questions during W09 to identify the source of confusion exhibited by students when presented with an application-based vector projection problem. After completing the same online vector analysis tutorial, students were asked to identify the formula that they would use to calculate the scalar projection of one vector onto another vector (Fig. 3a). This question was designed to investigate the approach taken by the student to solve a vector projection problem, without asking them to perform the mathematical operation. We observed that only $32 \%$ of the class was able to correctly identify the formula for calculating the scalar projection of one non-unit vector onto another non-unit vector (Fig. 10a), even after having just completed an interactive, for-credit review homework that included this material. Forty-five percent of the class believed that they would simply use a dot product to find the vector projection. After being given the correct formula, $74 \%$ of the class was able to use it to solve a subsequent vector projection problem, presented in a mathematical context similar to the online vector analysis tutorial (Fig. 10b).

a) "In order to calculate the scalar projection of a vector A onto a vector B, you would use:"

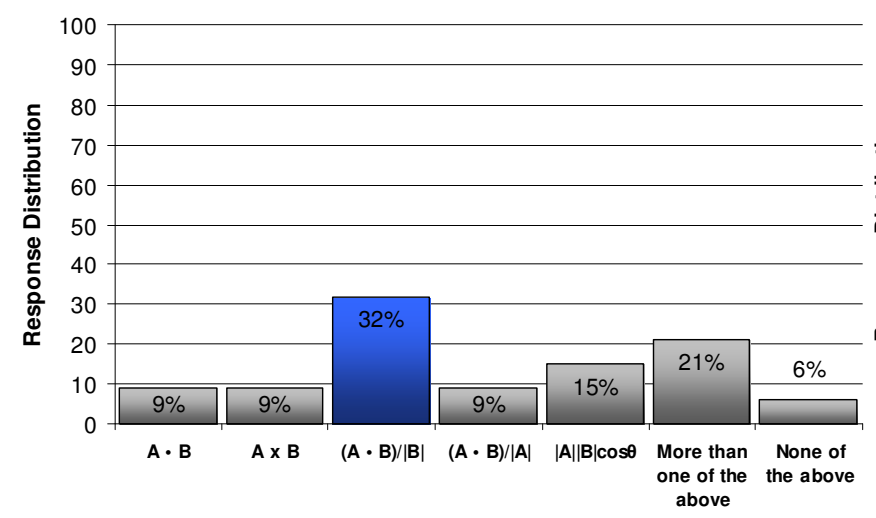

b) "The projection of vector B onto vector A is:"

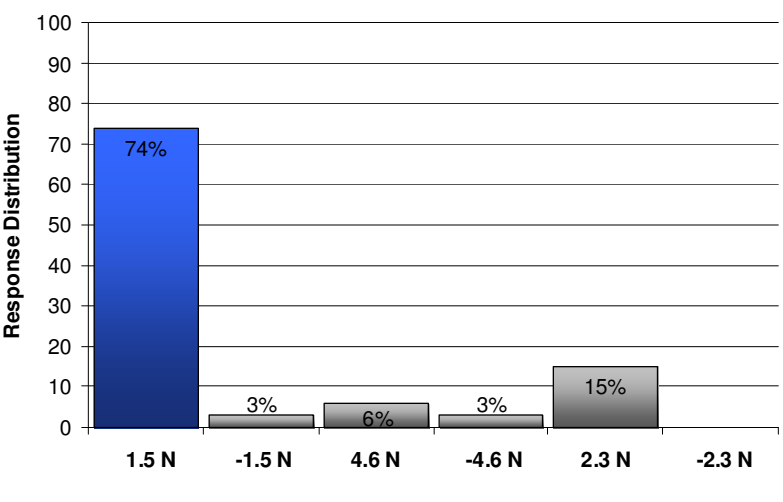

Figure 10: Response distribution of W09 PRS questions, shown as percent of total responses. Correct responses are shown in blue.

In the next class period, students were presented with a PRS question comparable to the W08 class (apart from re-wording the question, the same skills were assessed, Fig. 3c). Similar to W08, we observed that when a vector projection problem was embedded within a PRS story problem, only 35\% of the class responded correctly. Unlike W08 however, the most common mistake seemed to involve a misunderstanding concerning the quadrant that contained the vector $\vec{g}$ (Table 1). 


\subsubsection{Winter Quarter 2010 (W10)}

One class period after completing the vector analysis tutorial, W10 students were given a series of PRS questions similar to W09. According to the response distribution shown in Figure 11a, less than $50 \%$ of W10 students were able to identify the correct formula for calculating the scalar projection of one vector onto another vector, even after having just completed the vector analysis tutorial. Although $71 \%$ of W10 students were able to calculate a vector projection on the vector analysis post-test, only $38 \%$ of the class successfully demonstrated this skill in comparable PRS question (Fig. 11b). Furthermore, when asked to visualize the quadrant that contained the calculated projection, students responded with a considerable level of confusion (Fig. 11c shows an equal response distribution for each of the 4 possible answers).

Similar to W08 and W09, students in W10 were presented with a PRS story problem. The response distribution summarized in Table 1 shows that $44 \%$ of students were able to correctly identify and apply vector projection concepts to solve this story problem, compared to $35 \%$ in W09, and $20 \%$ in W08.

a) "In order to calculate the scalar projection of a vector A onto a vector B, you would use:"

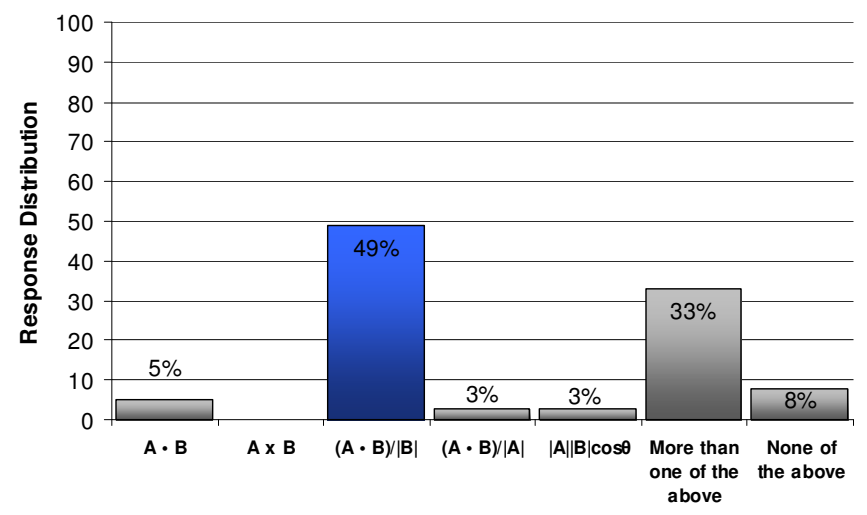

c)
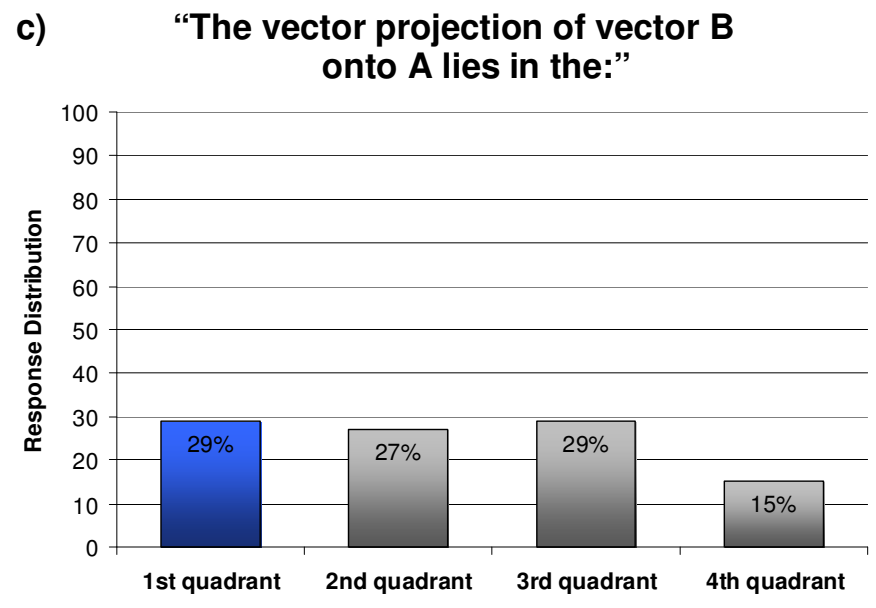

\section{b) "The projection of vector B onto} vector A is:"

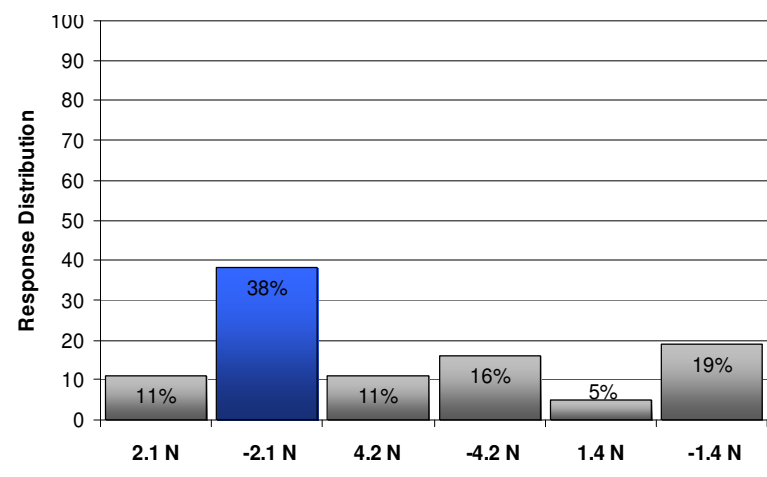

Figure 11: Response distribution of W10 PRS questions, shown as percent of total responses. Correct responses are shown in blue. 


\subsection{Application-Driven Homework}

Several weeks later and shortly before the midterm exam, W10 students were assigned an application-driven, "Reality Check" homework which required them to recognize a vector projection problem embedded within a story problem. Out of 43 students, only 11 students (26\%) attempted to calculate the projection of the applied load in the direction of the longitudinal axis of the radius. Of these 11 students, only 2 students (5\%) arrived at the correct answer. As part of the assignment, students were asked to rate their confidence in their analysis at the time they submitted their results: 1 student indicated that they were very confident that their analysis and interpretation were correct; 2 students thought that their analysis was correct, but were not sure how to interpret their results; 27 students indicated that they used things they learned from class to complete the analysis, but remained unsure if what they did was correct; and 12 indicated that they did the best they could, but did not know how to approach the problem, and were not confident in their answer. Overall, both performance and confidence in solving the application-driven homework assigned during W10 were low.

\subsection{Learning Outcome: Midterm Exam}

During W09, 61\% of students demonstrated mastery of vector projections on the midterm exam. Despite online review courseware and a series of diagnostic PRS questions, common mistakes on the W09 midterm exam included: mathematic mistakes, using the wrong formula (i.e., despite the use of non-unit vectors in the problem, students simply used a dot product to calculate the scalar projection of one vector onto another), and interpreting the solution as a vector, not a scalar. Even with the addition of the application-driven homework in W10, only 56\% of students were able to successfully answer a vector projection problem on the midterm exam, showing no improvement over W09. These results motivate further investigation into development methods designed to improve performance in this skill.

\section{Discussion and Conclusions}

For many first- and second-year engineering students, learning to transfer fundamental engineering concepts to problems encountered in the real world represents a significant challenge. Problem-solving strategies demand several upper-level cognitive processes, including application, analysis, synthesis, and evaluation ${ }^{16}$. In order to achieve these goals, engineering students must demonstrate basic skills in math and science; they must also recognize when to apply these skills. Unfortunately, the inability to apply fundamental concepts is a common obstacle for many students in gateway engineering courses. Accordingly, significant opportunities exist to improve achievement in these courses by shifting instructional focus toward basic skills remediation and problem-based learning activities.

It has been well documented that in various areas of engineering mechanics, students tend to struggle with applications in vector analysis, a prerequisite skill usually covered during the first year of most engineering curricula. In an effort to support applied skills in mechanics courses, vector analysis has become the focus of many dedicated remediation efforts, including in-class

review sessions and, more recently, computer-aided learning methods ${ }^{1,17-21}$. Compared to traditional instructional methods, computer-aided learning methods are often designed to be 
learner-centered, allowing students to proceed through material at their own pace while offering assistance and background material specific to the learner's needs. In addition, computer-aided learning methods allow students to practice in an active learning environment, which provides immediate (often diagnostic) formative feedback. Overall, several studies have shown that in a short period of time, computer-aided learning methods appear to help students better learn vector analysis and its applications ${ }^{20-22}$.

The data presented in this paper suggest that insufficient competency in prerequisite course material, specifically vector analysis, may hinder students' progress toward developing a meaningful understanding of concepts presented in introductory mechanics courses. In order to address this issue, we have incorporated several innovative teaching methods into our instruction base, including online courseware developed by the VaNTH ERC ${ }^{1}$ to review concepts in vector analysis, PRS to enhance formative assessment, and challenge-based homework assignments to emphasize the application of fundamental engineering skills in biomechanics.

A comparison between pre- and post-test results from W09 and W10 indicates that the online vector analysis tutorial used in this course was indeed able to improve students' performance of basic vector math, with the largest gains observed in vector multiplication and vector projections. These results agree with a similar evaluation study published by Rothney et $\mathrm{al}^{22}$. However, given the performance of the class on the follow-up PRS story problems in W08, W09, and W10, we believe that this online tutorial does not fully address underlying misconceptions involving the application of vector projections. Through the strategic use of PRS assessments, we have begun to unravel some of the hurdles that students face when presented with conceptual applications of vector algebra. After posing a PRS question during a W09 lecture, we observed that only 32\% of the class was able to correctly identify the formula for calculating the scalar projection of one non-unit vector onto another non-unit vector, even after having just completed an interactive, for-credit review homework that included this material. After being given the correct formula, $74 \%$ of the class was able to use it to solve a subsequent vector projection problem. Yet in the next class period, when a vector projection problem was embedded within a PRS story problem, only $35 \%$ responded correctly. While the exact percentages for the diagnostic and applied questions changed in W10, the trends were the same, and when students were given an out-ofcontext, applied problem, only 11 out of 43 even recognized the utility in calculating a vector projection to solve a problem which included phrases such as "the orthopaedic surgeon has concluded that the fracture occurred in response to a compressive load that was directed along the longitudinal axis of the radius". Experiences like these suggest to us that many students lack the ability to transfer basic skills when they are presented with an applied problem. By emphasizing the conceptual application of these skills in class, we have observed an increase in correct responses to story-based vector projection problems from $20 \%-44 \%$ (on the first appearance of such a problem in class, delivered via PRS) to $56 \%-61 \%$ on the midterm exam. Encouraged by these results, we continue to seek ways to expand the challenge-based learning methods, previously focused primarily on new course material, to emphasize competency in these basic skills.

\section{Acknowledgements:}

We would like to thank Stacy Klein, PhD from Vanderbilt University for sharing her VaNTH related course materials, including the PRS story problem question posed in our W08 class. 


\section{References}

1. Howard, L., Adaptive learning technologies for biomedical education. IEEE Engineering in Medicine and Biology Magazine, 2003. 22(4): p. 58-65.

2. Linsenmeier, R. A., What makes a biomedical engineer? IEEE Engineering in Medicine and Biology Magazine, 2003. 22(4): p. 32-38.

3. Gatchell, D. W., Linsenmeier, R. and Harris, T. R. Biomedical engineering key content survey-The 1st step in a delphi study to determine the core undergraduate BME curriculum. Proceedings of the American Society for Engineering Education Annual Conference and Exposition. 2004.

4. Gatchell, D. W. and Linsenmeier, R. Undergraduate biomedical engineering curricula: Recommendations from academia and industry. Proceedings of the Annual Meeting of the Institute of Biological Engineering. 2005.

5. Whitaker Biomedical Engineering Education Summit Meeting. 2000 (Accessed on Jan. 10, 2010). http://bmes.seas.wustl.edu/WhitakerArchives/summit/index.html.

6. Hung, C. and Sah, R. Biomechanics Curriculum. Whitaker Biomedical Engineering Education Summit Meeting. 2005.

7. Pandy, M. G., Petrosine, A. J., Barr, R. E., Tennant, L. and Seth, A. Design, implementation, and assessment of an HPL-inspired undergraduate course in biomechanics. Proceedings of the American Society for Engineering Education Annual Conference and Exposition. 2003.

8. Roselli, R. J. and Brophy, S. P. Movement from a taxonomy-driven strategy of instruction to a challengedriven strategy in teaching introductory biomechanics. Proceedings of the American Society for Engineering Education Annual Conference and Exposition. 2001.

9. Roselli, R. and Brophy, S., Effectiveness of challenge-based instruction in biomechanics. Journal of Engineering Education, 2006. 95: p. 311-324.

10. Bransford, J. D., Brown, A. L. and Cocking, R. R., How People Learn: Brain, Mind, Experience, and School. 1999, Washington, D.C.: National Academy Press.

11. Simpson, V. and Oliver, M., Electronic voting systems for lectures then and now: A comparison of research and practice. Australian Journal of Educational Technology, 2007. 23: p. 187-208.

12. Burnstein, R. A. and Lederman, L. M., Using wireless keypads in lecture classes. Physics Teacher, 2001. 39(1): p. 8-11.

13. Pazos, P., Linsenmeier, R. and Olds, S. Using technology to promote active learning in biomedical engineering. Proceedings of the American Society for Engineering Education Annual Conference and Exposition. 2007.

14. Roselli, R. J. and Brophy, S. P. Exploring an electronic polling system for the assessment of student progress in two biomedical engineering courses. Proceedings of the American Society for Engineering Education Annual Conference and Exposition. 2002.

15. Mazur, E., Peer Instruction: A User's Manual. 1997, New Jersey: Prentice Hall.

16. Nilson, L. B., Teaching At Its Best: A Research-Based Resource for College Instructors. 2003, Bolton, MA: Anker Publishing Company, Inc.

17. Rezaei, A., Jawaharlal, M., Kim, K. and Shih, A. On development of a hybrid vector statics course to reduce failure rate. Proceedings of the American Society of Engineering Education Annual Conference and Exposition. 2007.

18. Boulet, J. A. M., Lumsdaine, A. and Wasserman, J. F. The transition from textbook problems to realistic problems. Proceedings of the American Society of Engineering Education Annual Conference and Exposition. 2004.

19. Narayanan, G. V. Inclusion of hands-on interactive programs for teaching statics. Proceedings of the American Society of Engineering Education Annual Conference and Exposition. 2005.

20. Zaman, M. and Kukreti, A. R. Computer-based learning modules for rigid body mechanics. Proceedings of the American Society of Engineering Education Annual Conference and Exposition. 2000.

21. Cataloglu, E., Open source software in teaching physics: A case study on vector algebra and visual representations. The Turkish Online Journal of Educational Technology, 2006. 5(1): p. 68-74.

22. Rothney, M. P., Roselli, R. J. and Howard, L. Creation of an online vector addition tutorial: Exploring the advantages of providing diagnostic, multilevel feedback in basic skills remediation. Proceedings of the American Society of Engineering Education Annual Conference and Exposition. 2003. 